\title{
Protocols and Stability Analysis for Energy Harvesting TDMA Systems with/without Relaying
}

\author{
Ioannis Krikidis*, Gan Zheng ${ }^{\dagger}$, and Björn Ottersten ${ }^{\dagger}$ \\ *Department of Electrical and Computer Engineering, University of Cyprus, Cyprus \\ $\dagger$ Interdisciplinary Centre for Security, Reliability and Trust (SnT), University of Luxembourg \\ E-mail: krikidis@ucy.ac.cy, \{gan.zheng, bjorn.ottersten\}@uni.lu
}

\begin{abstract}
This paper deals with time-division multiple access (TDMA) in wireless networks with bursty data traffic and energy harvesting capabilities at the source nodes. Several TDMA-based transmission strategies that incorporate different levels of "assistance" for the energy-constrained sources are investigated and analyzed in terms of stable throughput. Specifically, we study the stability region for a TDMA policy with assistance of a) channel state information (CSI) at the sources, b) orthogonal relaying cooperation, c) cognitive radio relaying cooperation and d) fullduplex (FD) relaying cooperation. The transmission parameters associated with the proposed strategies are also optimized. We observe that CSI results in significant energy savings and enlarge the stability region of the network while FD cooperation, which exploits the multipacket reception characteristics of the channel, provides the best performance.
\end{abstract}

Index Terms-Energy harvesting, relay channel, cognitive radio, full-duplex relay, stability.

\section{INTRODUCTION}

In conventional power-constrained communication systems which are characterized by batteries, network lifetime maximization is a major concern and has been a hot research topic for decades [1]. The integration of the energy harvesting technology into communication systems is a powerful solution to the problem of network lifetime as terminals can harvest energy from the environment (solar, wind, electromagnetic waves etc) [2], [3]; energy harvesting is a significant technological step towards fully independent and autonomous networks. The energy harvesting concept has changed the definition of energy-efficiency in communication systems and the main question that designers have to address is the optimal management of the harvested energy.

Several papers in the literature deal with the above fundamental question from an information theoretic standpoint as well as from communication/networking perspectives. The capacity of a point-to-point communication system with energy harvesting capabilities is given in [4] for a fading Gaussian channel. The design of energy-harvesting based transmission policies that maximize throughput or minimize time delay is discussed in [5], [6] (and references therein) for different contexts. The problem of data scheduling for a slotted-based system with energy harvesting under a Markovian channel model is presented in [7]. However, the work in [8]-[11] is the most relevant to the problems studied in this paper. In [8], the authors analyze the stability region for a random multple-access network with energy-harvesting sources under a collision channel; this work is extended in [9] for a multipacket reception (MPR) channel. A cognitive radio network with an energy harvesting secondary node is modelled as an asymmetric random multiple access channel in [10] and analyzed in terms of stable throughput. On the other hand, the stability region for a basic orthogonal three-node cooperative system with energy harvesting capabilities at the source and the relay node is presented in [11].

In this paper we focus on the fundamental time-division multiple access (TDMA) channel with energy harvesting capabilities when the data traffic and the energy harvested are modeled as stochastic processes. Based on a simple network topology, we study several modifications of the conventional TDMA scheme that enable different techniques for assisting the energy harvesting sources. More specifically we investigate four TDMA schemes where the energy harvesting sources are assisted by a) a channel state information (CSI) at the transmitters which protects the sources from useless energy consumption, b) a half-duplex (HD) relay node where the relay accesses the channel in dedicated time slots, c) a cognitive radio (CR) relay node where the relay accesses the radio channel only when it becomes idle and d) a full-duplex (FD) relay node where the relay can simultaneously receive and transmit data packets. The investigated schemes are analyzed in terms of stable throughput and their stability region is derived in closed form. We show that FD relaying provides the largest stability region and is an attractive solution in order to assist/support TDMA energy harvesting systems. To the best of the authors' knowledge the investigated TDMA energyharvesting schemes are reported for first time in this paper.

The remainder of this paper is organized as follows. Section II introduces the system model and the basic assumptions for the data traffic and the energy harvested. Section III presents the investigated non-cooperative and cooperative protocols and gives their stability region. In section IV, the stability of the investigated schemes is quantified through numerical results and section $\mathrm{V}$ concludes the paper.

\section{SYSTEM MODEL}

We consider a simple network consisting of two sources $A, B$, a common relay $R$ and a common destination $D$. Time is considered to be slotted and the sources access the channel through a (randomized) TDMA scheduling scheme where $\omega_{i}$ denotes the probability that the $i$-th source (where 

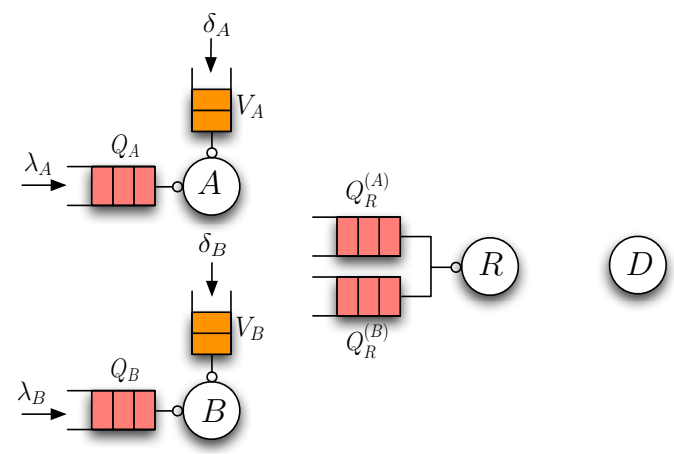

Fig. 1. The network model with/without cooperation between the two sources $A, B$ and the relay $R$.

$i \in\{A, B\})$ is allocated the whole time slot with $\omega_{i} \leq 1$ and $\omega_{A}+\omega_{B} \leq 1$; the transmission time for a packet is equal to one time slot. Each source has an infinite capacity buffer $Q_{i}$ for storing arriving data packets of fixed length and an infinite capacity battery ${ }^{1} V_{i}$ for storing harvested energy (energy packets of fixed length). The data packets and the harvested energy arrive at the users according to independent and stationary Bernoulli processes with mean $\lambda_{i}$ and $\delta_{i}$ (packets per slot), respectively, for the $i$-th user. We assume that one energy packet is consumed for each time slot either the source transmits or not. Although this assumption simplifies the analysis and allows closed-form expressions, it is also reasonable for some cases where the required energy to maintain the operation of the transmitter dominates its total energy consumption [12]. The relay node is connected to a constant power source (i.e., a base-station in a cellular architecture) and thus is not energy-constrained. It does not generate its own data traffic and is only used in order to assist the energy harvesting nodes $A, B$ by relaying their data packets. The cooperative strategy and the relay's channel access depends on the considered protocol and the technological characteristics of the relay node; Section III-B discusses some cooperative strategies with different capabilities at the relay node. In order to perform cooperation, the relay node is equipped with two infinite buffer queues $Q_{R}^{(i)}$ (one for each source). Fig. 1 schematically presents the system model.

Wireless links are characterized by the success probability of a packet, which is the probability that a packet can be decoded without errors. The channel allows probabilistic receptions of simultaneously transmitted packets (MPR channel [13]) and thus $f_{i, j}$ and $f_{i, j}^{I}$ (with $f_{i, j}>f_{i, j}^{I}$ ) denote the success probability without and with simultaneous transmission (interference), respectively, for the $i \rightarrow j$ link. The retransmission process is based on an Acknowledgement/NegativeAcknowledgement (ACK/NACK) mechanism, in which shortlength error-free packets are broadcast by the destinations over a separate narrow-band channel in order to inform the network

\footnotetext{
${ }^{1}$ It is a common assumption in the literature in order to study the stability region [5], [8], [10], [11] (it serves as a useful bound). In addition, if the energy buffer (battery) is large enough compared to packet size and energy consumed in a slot, this is a reasonable approximation [5].
}

of that packet's reception status.

The performance metric used in this work is the stable throughput region which refers to the union of all sets $\left(\lambda_{A}, \lambda_{B}\right)$ such that all queues in the system remain stable. $\mathrm{A}$ fundamental tool to characterize stability is Loynes' theorem [14], which states that if the arrival and service processes of a queue are jointly stationary, the queue is stable if and only if the average arrival rate is less than the average service rate.

\section{TDMA PROTOCOLS AND STABILITY REGION}

In this section we design different protocols for the TDMA channel considered and we derive their maximum stable throughput in closed-form. The investigated schemes are divided to non-cooperative and cooperative schemes.

\section{A. Non-cooperative protocols}

1) Conventional TDMA: The first protocol is the simplest one and is used as a baseline scheme. In conventional TDMA the sources $A$ and $B$ access the channel without any assistance and when the time slot is assigned to them. By Loynes' theorem, for a fixed $\left(\omega_{A}, \omega_{B}\right)$, the stability condition is defined by

$$
\lambda_{i}<\mu_{i}=\omega_{i} \mathbb{P}\left[V_{i} \neq 0\right] f_{i, D}=\omega_{i} \delta_{i} f_{i, D},
$$

where $\mathbb{P}\left[V_{i} \neq 0\right]=\delta_{i}$ as $V_{i}$ is modeled as a $\mathrm{M} / \mathrm{D} / 1$ system with arrival rate $\delta_{i}$ and service rate 1 [10]. If we take the union over all $\left(\omega_{A}, \omega_{B}\right)$ such as $\omega_{A}+\omega_{B} \leq 1$, we obtain the stability region which is

$$
\mathcal{R}_{\mathrm{TDMA}}=\left\{\left(\lambda_{A}, \lambda_{B}\right): \frac{\lambda_{A}}{\delta_{A} f_{A, D}}+\frac{\lambda_{B}}{\delta_{B} f_{B, D}}<1\right\} .
$$

2) TDMA with CSI: This protocol is an improvement of the conventional TDMA scheme and incorporates CSI knowledge at the sources. The available CSI is supported by an appropriate instantaneous feedback channel and can be used in order to optimize the TDMA transmissions. In order to study the CSI enhancement, we adopt a Gilbert Elliot model and thus all wireless channels are independent two-state discrete Markov chains [7]; the first state is defined as " $G$ " (good channel) and the second state is defined as " $B$ " (bad channel). The packet success probabilities and the channel steady state probabilities are denoted by $f_{i, D}^{(s t)}$ and $\pi_{i}^{(s t)}$, respectively, for the link $i \rightarrow D$ and the channel state $s t \in\{B, G\}$. In order to be consistent with the considered system model, we assume $f_{i, D}=\pi_{i}^{(G)} f_{i, D}^{(G)}+\pi_{i}^{(B)} f_{i, D}^{(B)}$. In this context, CSI refers to knowledge of the channel steady state at the sources and is exploited as follows:

- If the channel is in state ' $\mathrm{G}$ ', the $i$-th source transmits with probability 1 .

- If the channel is in state 'B', the $i$-th source transmits with probability $p_{i}$.

By Loynes' theorem, for a fixed $\left(\omega_{A}, \omega_{B}\right)$, the stability condition is defined by

$$
\lambda_{i}<\mu_{i}=\omega_{i} \mathbb{P}\left[V_{i} \neq 0\right]\left(\pi_{i}^{(G)} f_{i, D}^{(G)}+\pi_{i}^{(B)} p_{i} f_{i, D}^{(B)}\right),
$$


where

$$
\mathbb{P}\left[V_{i} \neq 0\right]=\min \left[1, \frac{\delta_{i}}{\pi_{i}^{(G)}+\pi_{i}^{(B)} p_{i}}\right] .
$$

The stability region for specific values of $\left(p_{A}, p_{B}\right)$ is given by the union over all $\left(\omega_{A}, \omega_{B}\right)$ and can be written as

$$
\begin{gathered}
\frac{\lambda_{A}\left(\pi_{A}^{(G)}+\pi_{A}^{(B)} p_{A}\right)}{\min \left[\delta_{A}, \pi_{A}^{(G)}+\pi_{A}^{(B)} p_{A}\right]\left(\pi_{A}^{(G)} f_{A, D}^{(G)}+\pi_{A}^{(B)} p_{A} f_{A, D}^{(B)}\right)} \\
+\frac{\lambda_{B}\left(\pi_{B}^{(G)}+\pi_{B}^{(B)} p_{B}\right)}{\min \left[\delta_{B}, \pi_{B}^{(G)}+\pi_{B}^{(B)} p_{B}\right]\left(\pi_{B}^{(G)} f_{B, D}^{(G)}+\pi_{B}^{(B)} p_{B} f_{B, D}^{(B)}\right)}<1 .
\end{gathered}
$$

The stability region is the union of all such regions as $\left(p_{A}, p_{B}\right)$ varies over $[0,1]^{2}$ and is written as

$$
\begin{aligned}
\mathcal{R}_{\mathrm{TDMA}-\mathrm{CSI}}= & \left\{\left(\lambda_{A}, \lambda_{B}\right): \frac{\lambda_{A}}{\pi_{A}^{(G)}\left(f_{A, D}^{(G)}-f_{A, D}^{(B)}\right)+\delta_{A} f_{A, D}^{(B)}}\right. \\
& \left.+\frac{\lambda_{B}}{\pi_{B}^{(G)}\left(f_{B, D}^{(G)}-f_{B, D}^{(B)}\right)+\delta_{B} f_{B, D}^{(B)}}<1\right\} .
\end{aligned}
$$

3) TDMA Vs TDMA-CSI: As can be seen from (2), (6), the stability region for both non-cooperative TDMA protocols is bounded by a straight line. Therefore, in order to compare their stability regions, it is enough to compare the intersection of the two stability regions with the axes. The intersections points for both TDMA schemes are given by

$$
\begin{aligned}
& \lambda_{i}^{*}(\text { TDMA })=\delta_{i} f_{i, D}=\delta_{i}\left[\pi_{i}^{(G)}\left(f_{i, D}^{(G)}-f_{i, D}^{(B)}\right)+f_{i, D}^{(B)}\right], \\
& \lambda_{i}^{*}(\text { TDMA-CSI })=\pi_{i}^{(G)}\left(f_{i, D}^{(G)}-f_{i, D}^{(B)}\right)+\delta_{i} f_{i, D}^{(B)} .
\end{aligned}
$$

We can see that $\lambda_{i}^{*}$ (TDMA) $\leq \lambda_{i}^{*}$ (TDMA-CSI) and therefore $\mathcal{R}($ TDMA $) \subseteq \mathcal{R}$ (TDMA-CSI); the two regions coincides for $\delta_{i}=1$

\section{B. Cooperative schemes}

In order to simplify the presentation, the cooperative schemes are analyzed for the non-CSI case; CSI can be exploited in a similar way to Section III-A2 in order to further improve the performance.

1) TDMA with orthogonal relaying: In this protocol, the two sources are assisted by a HD relay node which is connected to a constant power source; the HD limitation means that the relay node cannot receive and transmit data simultaneously. The relay node accesses the channel in dedicated time slots (orthogonal relaying) and it serves one relaying queue at the time. The probability that the $i$-th source accesses the channel as well as the probability that the relay node assists the $i$-th source are equal to $\omega_{i} / 2$. The cooperative strategy that is considered is stated as follows: the $i$-th source transmits a packet with a probability $\omega_{i} / 2$ given that its battery is not empty; if the destination can successfully decode it, $D$ feeds an ACK signal and the packet is removed from $Q_{i}$; otherwise, if $D$ does not decode the packet but $R$ decode it, $R$ feeds an
ACK signal and the packet is placed at the $Q_{R}^{(i)}$ queue for relaying transmission while is removed from $Q_{i}$; if neither $R$ nor $D$ decode the packet, the packet remains at $Q_{i}$ for retransmission. The relay node serves the $Q_{R}^{(i)}$ queue with a probability $\omega_{i} / 2$; if the packet is successfully decoded at the destination, $D$ transmits an ACK signal and the packet is removed from $Q_{R}^{(i)}$; otherwise it remains in $Q_{R}^{(i)}$ for rerelaying.

To ensure that the system is stable, all the queues have to be stable. The stability condition for the source queue $Q_{i}$ requires

$$
\lambda_{i}<\frac{1}{2} \omega_{i} \mathbb{P}\left[V_{i} \neq 0\right] g_{i}=\frac{1}{2} \omega_{i} \delta_{i} g_{i},
$$

where $g_{i}=f_{i, D}+g_{i}^{\prime}$ and $g_{i}^{\prime}=\left(1-f_{i, D}\right) f_{i, R}$. The arrival rate and the departure rate for the $Q_{R}^{(i)}$ queue are given by

where

$$
\begin{aligned}
\lambda_{R}^{i} & =\frac{1}{2} \omega_{i} \mathbb{P}\left[V_{i} \neq 0, Q_{i} \neq 0\right] g_{i}^{\prime}, \\
\mu_{R}^{i} & =\frac{1}{2} \omega_{i} f_{R, D},
\end{aligned}
$$

$$
\mathbb{P}\left[V_{i} \neq 0, Q_{i} \neq 0\right]=\frac{2 \lambda_{i}}{\omega_{i} g_{i}} .
$$

The above expression uses a fundamental property of the stable systems that the arrival rate is equal to the departure rate $[8$, App. A]. The stability condition of the relaying queue $Q_{R}^{(i)}$ requires

$$
\lambda_{R}^{i}<\mu_{R}^{i} \Rightarrow \lambda_{i}<\frac{1}{2} \frac{\omega_{i} f_{R, D} g_{i}}{g_{i}^{\prime}} .
$$

By taking the union over all feasible $\left(\omega_{A}, \omega_{B}\right)$, the stability region is given by

$$
\begin{aligned}
& \mathcal{R}_{\text {TDMA-HD }}=\left\{\left(\lambda_{A}, \lambda_{B}\right): \frac{\lambda_{A}}{\min \left[\delta_{A}, \frac{f_{R, D}}{g_{A}^{\prime}}\right] g_{A}}\right. \\
& \left.+\frac{\lambda_{B}}{\min \left[\delta_{B}, \frac{f_{R, D}}{g_{B}^{\prime}}\right] g_{B}}<\frac{1}{2}\right\} .
\end{aligned}
$$

2) TDMA with cognitive cooperation: In a TDMA scheme with cognitive cooperation, the relay node behaves as a CR node and accesses the channel only when the sources are inactive without requiring dedicated channel resources [15]; the slot access is similar to the conventional TDMA policy and the spectrum sensing is assumed to be perfect. The cooperative strategy follows the same principles as the TDMAHD scheme, except for the relaying transmission. More specifically, the relay node senses the channel and serves the $Q_{R}^{(i)}$ queue when the slot is assigned to the $i$-th source and the $i$-th source is inactive (it has an empty battery or an empty data queue $\left.Q_{i}\right)$. To ensure that the system is stable, both source queues and the relaying queues have to be stable. By Loynes' theorem the stability condition for the source queue requires

$$
\lambda_{i}<\mu_{i}=\omega_{i} \mathbb{P}\left[V_{i} \neq 0\right] g_{i}=\omega_{i} \delta_{i} g_{i} .
$$


The arrival and the departure rate for the relaying queue $Q_{R}^{(i)}$ are calculated as follows

$$
\begin{aligned}
& \lambda_{R}^{i}=\omega_{i} \mathbb{P}\left[V_{i} \neq 0, Q_{i} \neq 0\right] g_{i}^{\prime}, \\
& \mu_{R}^{i}=\omega_{i}\left(1-\mathbb{P}\left[V_{i} \neq 0, Q_{i} \neq 0\right]\right) f_{R, D}
\end{aligned}
$$

By using the same argument with the previous scheme, the probability that the $i$-th source is active is equal to

$$
\mathbb{P}\left[V_{i} \neq 0, Q_{i} \neq 0\right]=\frac{\lambda_{i}}{\omega_{i} g_{i}},
$$

and therefore the stability condition for the relaying queue $Q_{R}^{(i)}$ gives

$$
\lambda_{R}^{i}<\mu_{R}^{i} \Rightarrow \lambda_{i}<\omega_{i} \frac{f_{R, D} g_{i}}{g_{i}^{\prime}+f_{R, D}}
$$

By combining (14), (18), the stability region for fixed $\left(\omega_{A}, \omega_{B}\right)$ is written as

$$
\lambda_{i}<\omega_{i} g_{i} \min \left[\delta_{i}, \frac{f_{R, D}}{g_{i}^{\prime}+f_{R, D}}\right],
$$

and by taking the union over all feasible $\left(\omega_{A}, \omega_{B}\right)$ we have stability region of the system

$$
\begin{aligned}
\mathcal{R}_{\text {TDMA-Cg }}=\left\{\left(\lambda_{A}, \lambda_{B}\right): \frac{\lambda_{A}}{\min \left[\delta_{A}, \frac{f_{R, D}}{g_{A}^{\prime}+f_{R, D}}\right] g_{A}}\right. \\
\left.+\frac{\lambda_{B}}{\min \left[\delta_{B}, \frac{f_{R, D}}{g_{B}^{\prime}+f_{R, D}}\right] g_{B}}<1\right\} .
\end{aligned}
$$

3) TDMA with full-duplex relaying: This protocol incorporates a FD relay for assisting with the source transmissions; FD means that the relay node can simultaneously ${ }^{2}$ transmit and receive data. The full-duplex operation is assumed to be ideal and therefore no-loop interference affects the relay reception. The cooperative strategy is similar to the previous cooperative schemes except of the behavior of the relay node. More specifically, the relay node serves the $Q_{R}^{(i)}$ when the time slot is assigned to the $i$-th source as follows:

- It transmits with probability 1 when the $i$-th source is inactive.

- It transmits with probability $p_{i}$ when the $i$-th source is active.

In case that both the $i$-th source and the relay node access the channel, the destination node has MPR capabilities and therefore can decode both packets with non-zero probability. On the other hand, due to the perfect full-duplex operation, simultaneous transmission does not affect the source-relay link. It is worth noting that for the case of simultaneous transmissions, the destination adopts a 2-bit ACK/NACK mechanism in order to inform the transmitters about the

\footnotetext{
${ }^{2}$ Recent advances on antennas technology and signal processing make FD feasible i.e., [16]
}

status of both transmitted packets. The stability of the system involves an interaction between the $Q_{i}$ and $Q_{R}^{(i)}$ queues that makes the analysis difficult. In order to solve this problem and characterize the stability of the system, we use the stochastic dominance approach introduced in [17]. We construct a dominant system $\mathcal{M}$ where the relay node transmits dummy packets when the serving relaying queue is empty; this assumption decouples the interaction between the $Q_{i}$ and $Q_{R}^{(i)}$ queues. In the following discussion we characterize the stability of the dominant system $\mathcal{M}$.

The stability condition for the source queue $Q_{i}$ requires

$$
\begin{aligned}
\lambda_{i} & <\omega_{i} \mathbb{P}\left[V_{i} \neq 0\right]\left(p_{i} h_{i}+\left(1-p_{i}\right) g_{i}\right) \\
& =\omega_{i} \delta_{i}\left(p_{i} h_{i}+\left(1-p_{i}\right) g_{i}\right),
\end{aligned}
$$

where $h_{i}=f_{i, D}^{I}+h_{i}^{\prime}$ and $h_{i}^{\prime}=\left(1-f_{i, D}^{I}\right) f_{i, R}$. The arrival and the departure rate for the relaying queue $Q_{R}^{(i)}$ are equal to

$$
\begin{array}{rl}
\lambda_{R}^{i}=\omega_{i} & \mathbb{P}\left[V_{i} \neq 0, Q_{i} \neq 0\right]\left[p_{i} h_{i}^{\prime}+\left(1-p_{i}\right) g_{i}^{\prime}\right] \\
\mu_{R}^{i}=\omega_{i}\left[\mathbb{P}\left[V_{i} \neq 0, Q_{i} \neq 0\right] p_{i} f_{R, D}^{I}\right. \\
\left.\quad+\left(1-\mathbb{P}\left[V_{i} \neq 0, Q_{i} \neq 0\right]\right) f_{R, D}\right]
\end{array}
$$

where

$$
\mathbb{P}\left[V_{i} \neq 0, Q_{i} \neq 0\right]=\frac{\lambda_{i}}{\omega_{1}\left[p_{i} h_{i}+\left(1-p_{i}\right) g_{i}\right]} .
$$

By Loynes' theorem the stability conditions for the relaying queue $Q_{R}^{i}$ require

$$
\lambda_{R}^{i}<\mu_{R}^{i} \Rightarrow \lambda_{i}<\omega_{i} \frac{\left[p_{i} h_{i}+\left(1-p_{i}\right) g_{i}\right] f_{R, D}}{p_{i}\left(h_{i}^{\prime}-f_{R, D}^{I}\right)+\left(1-p_{i}\right) g_{i}^{\prime}+f_{R, D}} .
$$

For a fixed $\left(p_{A}, p_{B}\right)$ the stability region is the intersection of the stability regions given by (21), (24) which gives

$$
\begin{aligned}
\lambda_{i}< & \omega_{i}\left[p_{i} h_{i}+\left(1-p_{i}\right) g_{i}\right] \\
& \times \min \left[\delta_{i}, \frac{f_{R, D}}{p_{i}\left(h_{i}^{\prime}-f_{R, D}^{I}\right)+\left(1-p_{i}\right) g_{i}^{\prime}+f_{R, D}}\right] .
\end{aligned}
$$

By taking the union over all $\left(p_{A}, p_{B}\right)$, the stability region of the system is summarized as follows:

$$
\mathcal{R}_{\mathrm{TDMA}-\mathrm{FD}}=\left\{\left(\lambda_{A}, \lambda_{B}\right): \frac{\lambda_{A}}{Y_{A}}+\frac{\lambda_{B}}{Y_{B}}<1\right\}
$$

where

$$
Y_{i}=\left\{\begin{array}{l}
g_{i} \delta_{i}, \quad \text { If } \mathbb{E}_{i_{0}} \\
\max \left[\frac{g_{i} f_{R, D}}{g_{i}^{\prime}+f_{R, D}},\left[p_{i}^{*} h_{i}+\left(1-p_{i}^{*}\right) g_{i}\right] \delta_{i}\right], \text { If } \mathbb{E}_{i_{1}} \\
{\left[p_{i}^{*} h_{i}+\left(1-p_{i}^{*}\right) g_{i}\right] \delta_{i}, \text { If } \mathbb{E}_{i_{2}}} \\
\min \left[g_{i} \delta_{i}, \frac{g_{i} f_{R, D}}{g_{i}^{\prime}+f_{R, D}}\right], \text { If } \mathbb{E}_{i_{3}}
\end{array}\right.
$$


with

$$
\begin{aligned}
& \Gamma_{i}=h_{i}^{\prime}-g_{i}^{\prime}, \\
& \Theta_{i}=\frac{g_{i}\left(h_{i}^{\prime}+f_{R, D}\right)-h_{i}\left(g_{i}^{\prime}+f_{R, D}\right)}{g_{i}}, \\
& p_{i}^{*}=\frac{f_{R, D}-\delta_{i}\left[g_{i}+f_{R, D}\right]}{\delta_{i}\left(\Gamma_{i}-f_{R, D}^{I}\right)}, \\
& \mathbb{E}_{i_{0}}=\left\{f_{R, D}^{I}<\Gamma_{i} \bigcap p_{i}^{*} \in\left[\begin{array}{ll}
0 & 1
\end{array}\right]\right\}, \\
& \mathbb{E}_{i_{1}}=\left\{\Theta_{i} \geq f_{R, D}^{I}>\Gamma_{i} \bigcap p_{i}^{*} \in\left[\begin{array}{ll}
0 & 1
\end{array}\right],\right. \\
& \mathbb{E}_{i_{2}}=\left\{f_{R, D}^{I}>\Theta_{i} \bigcap p_{i}^{*} \in\left[\begin{array}{ll}
0 & 1
\end{array}\right],,\right. \\
& \mathbb{E}_{i_{3}}=\left\{f_{R, D}^{I}=\Gamma_{i} \bigcup p_{i}^{*} \notin\left[\begin{array}{ll}
0 & 1
\end{array}\right]\right\} .
\end{aligned}
$$

Proof: see Appendix.

The above stability conditions that characterize the dominant system $\mathcal{M}$ are necessary and sufficient conditions for the stability of the original system. More specifically, the queues of the dominant system are always larger in size than those of the original system given that they are initialized to the same value; therefore, if the dominant system is stable for a $\lambda_{i}$ then the original system will be also stable (sufficient condition). On the other hand, if for a $\lambda_{i}$ in the dominant system the relaying queue $Q_{R}^{(i)}$ saturates, the relay node will not transmit dummy packets and the two systems (original and dominant) become indistinguishable (necessary condition).

4) TDMA-HD Vs TDMA-Cg Vs TDMA-FD: By using the same arguments with Section III-A3 a comparison of the above cooperative protocols requires the computation of the cross-over points of the stability region with the axes and it can be seen that $\lambda_{i}^{*}$ (TDMA-HD) $\leq \lambda_{i}^{*}$ (TDMA-Cg) $\leq$ $\lambda_{i}^{*}$ (TDMA-FD); therefore $\mathcal{R}_{\text {TDMA-HD }} \subseteq \mathcal{R}_{\text {TDMA-Cg }} \subseteq$ $\mathcal{R}_{\text {TDMA-FD }}$

\section{NUMERICAL RESULTS}

In this section we numerically evaluate the stability expressions given in the previous theoretical development. Fig. 2 plots the stability region of the investigated TDMA schemes for a symmetric set-up with $f_{i, D}=0.3, \delta_{i}=0.7, \pi_{i}^{(G)}=0.6$, $f_{i, D}^{(B)}=0.15, f_{i, R}=f_{R, D}=0.8, f_{i, D}^{I}=0.1$ and $f_{R, D}^{I}=0.5$. The first important observation is that CSI knowledge improves the stability region of the conventional TDMA scheme in an energy harvesting context. The exploitation of CSI results in a more efficient use of the energy harvested which enlarges the stable throughput of the system (it avoids wasteful energy consumption when the channel is poor). On the other hand, it can be seen that relaying assistance significantly improves the stability of the system. For the considered values of the success probabilities, the relay node offers a robust alternative end-toend link and thus all the cooperative schemes outperform both non-cooperative schemes. The TDMA-HD policy results in the smallest stable throughput (among the cooperative schemes) due to the HD constraint and the associated orthogonal channel access of the relay node. As for the TDMA-Cg scheme, it can be seen that it outperforms TDMA-HD as the relay node access the channel opportunistically without dedicated channel resources. The TDMA-FD policy behaves like TDMA-Cg and in addition exploits the MPR capability of the wireless channel; therefore the stability region of the TDMA-Cg is contained inside TDMA-FD. Therefore, full-duplex relay operation is introduced as an efficient technique for supporting systems with energy harvesting limitations.

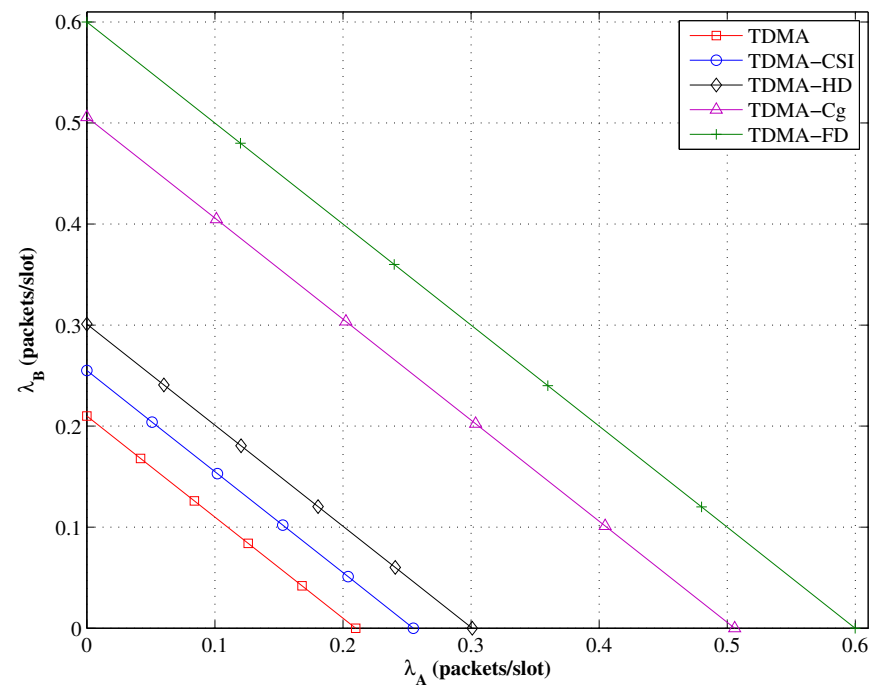

Fig. 2. Stability region for the investigated TDMA protocols; $f_{i, D}=0.3$, $\delta_{i}=0.7, \pi_{i}^{(G)}=0.6, f_{i, D}^{(B)}=0.15, f_{i, R}=f_{R, D}=0.8, f_{i, D}^{I}=0.1$ and $f_{R, D}^{I}=0.5$.

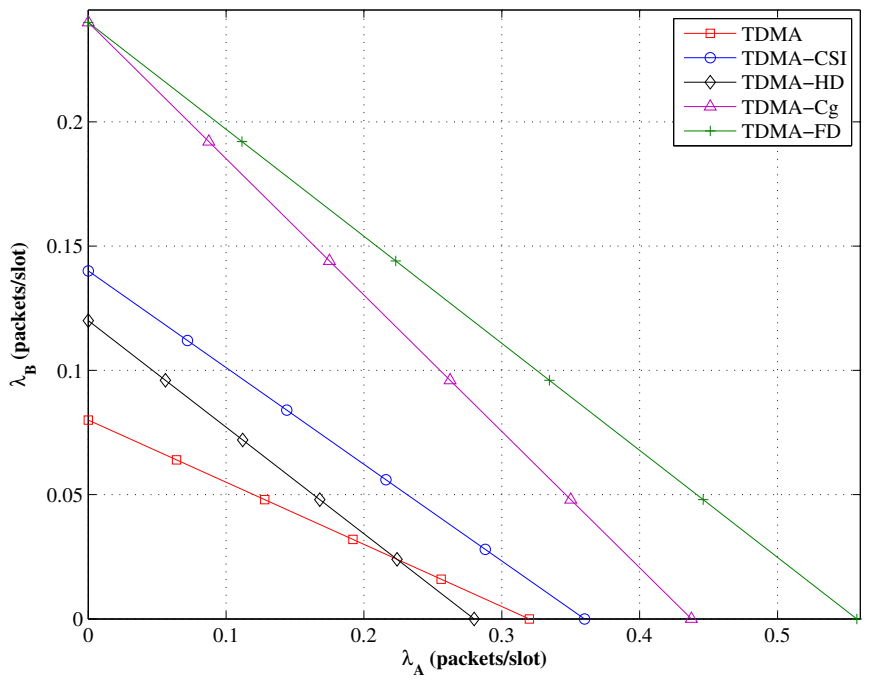

Fig. 3. Stability region for the investigated TDMA protocols; $f_{A, D}=0.4$, $f_{B, D}=0.2, \delta_{A}=0.8, \delta_{B}=0.4, \pi_{i}^{(G)}=0.6, f_{A, D}^{(B)}=0.2, f_{B, D}^{(B)}=0.1$, $f_{i, R}=f_{R, D}=0.5, f_{A, D}^{I}=0.3, f_{B, D}^{I}=0.1$ and $f_{R, D}^{I}=0.4$

Fig. 3 assumes an asymmetric simulation set-up with $f_{A, D}=0.4, f_{B, D}=0.2, \delta_{A}=0.8, \delta_{B}=0.4, \pi_{i}^{(G)}=0.6$, $f_{A, D}^{(B)}=0.2, f_{B, D}^{(B)}=0.1, f_{i, R}=f_{R, D}=0.5, f_{A, D}^{I}=0.3$, $f_{B, D}^{I}=0.1$ and $f_{R, D}^{I}=0.4$ (better channel and energy conditions for source $A$ ). Due to the asymmetry of the system parameters, the bounds of the stability regions are not parallel 
lines. It can be seen that the TDMA-CSI scheme outperforms the TDMA-HD scheme and thus for the considered channel conditions, CSI knowledge is more useful than allocating dedicated slots to an orthogonal relay. In addition, Fig. 3 shows that the conventional TDMA gives a higher maximum throughput than TDMA-HD for source $A$ due to the strong direct link $A \rightarrow D$. Finally, it can be observed that the FD cooperation provides the largest stability region and contains the stability region of the CR cooperation.

\section{CONCLUSION}

This paper has dealt with the TDMA policy in energy harvesting systems where data arrivals and energy harvested are modelled as stochastic processes. Several TDMA protocols that enable different techniques for source assistance have been investigated and analyzed in terms of stable throughput. More specifically, we studied several modifications of the conventional TDMA policy that incorporate a) CSI knowledge at the transmitters b) a HD relay, c) a CR relay and d) a FD relay. We have proved that CSI significantly improves the stability of the system as it ensures energy savings while the FD relaying provides the largest stability region and is an attractive solution for systems with energy harvesting constraints. The extension of the proposed schemes to more realistic network scenarios with CR sensing errors and FD loop-interference will be considered for future work.

\section{APPENDIX}

Based on (25) we examine the following cases: Case 1:

$$
\begin{aligned}
& \delta_{i} \leq \frac{f_{R, D}}{p_{i}\left(h_{i}^{\prime}-f_{R, D}^{I}\right)+\left(1-p_{i}\right) g_{i}^{\prime}+f_{R, D}} \\
& \Rightarrow p_{i}\left(\Gamma_{i}-f_{R, D}^{I}\right) \leq \frac{f_{R, D}-\delta_{i}\left(g_{i}^{\prime}+f_{R, D}\right)}{\delta_{i}}
\end{aligned}
$$

We examine the following subcases:

$\left(a_{1}\right)$ If $f_{R, D}^{I}<\Gamma_{i}$ and $p_{i}^{*} \in[01]$, (35) gives $p_{i} \leq p_{i}^{*}$ and thus $p_{i}=0$ maximizes the stability region given that the function $y_{1}\left(p_{i}\right)=p_{i} h_{i}+\left(1-p_{i}\right) g_{i}$ is a decreasing function of $p_{i}$.

$\left(b_{1}\right)$ If $f_{R, D}^{I}>\Gamma_{i}$ and $p_{i}^{*} \in[01]$, (35) gives $p_{i} \geq p_{i}^{*}$ and thus $p_{i}=p_{i}^{*}$ maximizes the stability region for the same reason with $\left(a_{1}\right)$.

Case 2:

$$
\begin{gathered}
\delta_{i}>\frac{f_{R, D}}{p_{i}\left(h_{i}^{\prime}-f_{R, D}^{I}\right)+\left(1-p_{i}\right) g_{i}^{\prime}+f_{R, D}} \\
\Rightarrow p_{i}\left(\Gamma_{i}-f_{R, D}^{I}\right)>\frac{f_{R, D}-\delta_{i}\left(g_{i}^{\prime}+f_{R, D}\right)}{\delta_{i}}
\end{gathered}
$$

We examine the following subcases:

$\left(a_{2}\right)$ If $f_{R, D}^{I}<\Gamma_{i}$ and $p_{i}^{*} \in[01]$, (36) gives $p_{i}>p_{i}^{*}$ and thus $p_{i}=p_{i}^{*}$ maximizes the stability region given that the function $y_{2}\left(p_{i}\right)=\left[p_{i} h_{i}+\left(1-p_{i}\right) g_{i}\right] /\left[p_{i}\left(h_{i}^{\prime}-f_{R, D}^{I}\right)+\left(1-p_{i}\right) g_{i}^{\prime}+f_{R, D}\right]$ is a decreasing function of $p_{i}$ (it can be shown that the first derivative is always negative $\left.y_{2}\left(p_{i}\right)^{\prime}<0\right)$. $\left(b_{2}\right)$ If $\Theta_{i} \geq f_{R, D}^{I}>\Gamma_{i}$ and $p_{i}^{*} \in\left[\begin{array}{ll}0 & 1\end{array}\right]$, (36) gives $p_{i}<p_{i}^{*}$ and thus $p_{i}=0$ maximizes the stability region for the same reason with $\left(a_{2}\right)$.

(c) If $f_{R, D}^{I}>\Theta_{i}$ and $p_{i}^{*} \in[01]$, (36) gives $p_{i}<p_{i}^{*}$ and thus $p_{i}=p_{i}^{*}$ maximizes the stability region given that the function $y_{2}\left(p_{i}\right)$ becomes increasing function in this case (its first derivation is always positive $y_{2}\left(p_{i}\right)^{\prime}>0$ ).

\section{Case 3:}

If $f_{i, D}^{I}=\Gamma_{i}$ or $p_{i}^{*} \notin\left[\begin{array}{ll}0 & 1\end{array}\right]$ then $p_{i}=0$ maximizes the stability region as both functions $y_{1}\left(p_{i}\right)$ and $y_{2}\left(p_{i}\right)$ are decreasing functions of $p_{i}$.

The combination of the above cases gives the stability region in (26).

\section{REFERENCES}

[1] L. V. Hoesel, T. Nieberg, W. Jian, and P. J. M. Havinga, "Prolonging the lifetime of wireless sensor networks by cross-layer interaction," IEEE Wireless Commun., vol. 11, pp. 78-86, Dec. 2004.

[2] L. Mateu and F. Moll, "Review of energy harvesting techniques and applications for microelectronics," in Proc. SPIE Circuits and Syst. II, vol. 5837, pp. 359-373, 2005.

[3] C. Alippi and C. Galperti, "An adaptive system for optimal solar energy harvesting in wireless sensor network nodes," IEEE Trans. Circ. Syst., vol. 55, pp. 1742-1750, July 2008.

[4] R. Rajesh, V. Sharma, and P. Viswanath, "Capacity of fading gaussian channel with an energy harvesting sensor node," in Proc. IEEE Global Commun. Conf., Houston, USA, Dec. 2011, pp. 1-6.

[5] V. Sharma, U. Mukherji, V. Joseph, and S. Gupta, "Optimal energy management policies for energy harvesting sensor nodes," IEEE Trans. Wireless Commun., vol. 9, pp. 1326-1336, Apr. 2010.

[6] O. Ozel, K. Tutuncuoglu, J. Yang, S. Ulukus, and A. Yener, "Transmission with energy harvesting nodes in fading wireless channels: optimal policies," IEEE J. Selec. Areas Commun., vol. 29, pp. 1732-1743, Sept. 2011.

[7] M. Kashef and A. Ephremides, "Optimal scheduling for energy harvesting sources on time varying wireless channels," in Allerton Conf. Commun. Cont. Comp., Urbana, USA, Sept. 2011, pp. 712-718.

[8] J. Jeon and A. Ephremides, "The stability region of random multiple access under stochastic energy harvesting," in Proc. IEEE Int. Symp. Inf. Theory, Saint Petersburg, Russia, July 2011, pp. 1796-1800.

[9] J. Jeon and A. Ephremides, "On the stability of random multiple access with stochastic energy harvesting," IEEE Trans. Inf. Theory, submitted for publication, Dec. 2011.

[10] N. Pappas, J. Jeon, A. Ephremides, and A. Traganitis, "Optimal utilization of a cognitive shared channel with a rechargeable primary source node," in Proc. IEEE Inf. Theory Work., Paraty, Brazil, Oct. 2011, pp. 598-602.

[11] I. Krikidis, T. Charalambous, and J. S. Thompson, "Stability analysis and power optimization for energy harvesting cooperative networks," IEEE Sign. Proc. Lett., vol. 19, pp. 20-23, Jan. 2012.

[12] H. Karl, "An overview of energy-efficiency techniques for mobile communication systems," Telecommunication Networks Group, Technical University Berlin, Tech. Rep. TKN-03-XXX, September 2003.

[13] V. Naware, G. Mergen, and L. Tong, "Stability and delay of finite-user slotted Aloha with multipacket reception," IEEE Trans. Inf. Theory, vol. 51, pp. 2636-2656, July 2005.

[14] R. Loynes, "The stability of a queue with non-interdependent interarrival and service times," in Proc. Camb. Phylos. Soc., vol. 58, pp. 497-520, 1962.

[15] A. K. Sadek, K. J. R. Liu, and A. Ephremides, "Cognitive multiple access via cooperation: protocol design and performance analysis," IEEE Trans. Inform. Theory, vol. 53, pp. 3677-3696, Oct. 2007.

[16] J. Choi, J. Mayank, S. Kannan, L. Philip, and K. Sachin, "Achieving single channel, full duplex wireless communications," in Proc. Int. Conf. Mob. Comp. Netw. Chicago, USA, Sept. 2010, pp. 1-12.

[17] R. Rao and A. Ephremides, "On the stability of interacting queues in a multiple-access system," IEEE Trans. Inf. Theory, vol. 34, pp. 918-930, Sept. 1988. 